Article

\title{
Efficient Near-Field Analysis of the Electromagnetic Scattering Based on the Dirichlet-to-Neumann Map
}

\author{
Antea M. Perrotta ${ }^{1}$, Antonio Maffucci ${ }^{1,2}{ }^{\mathbb{D}}$, Salvatore Ventre ${ }^{1}$ and Antonello Tamburrino ${ }^{1,3, * \mathbb{D}}$ \\ 1 Department of Electrical and Information Engineering, University of Cassino and Southern Lazio, \\ 03043 Cassino, Italy; a.perrotta@unicas.it (A.M.P.); maffucci@unicas.it (A.M.); ventre@unicas.it (S.V.) \\ 2 Nanotechnology Lab., INFN, National Institute of Nuclear Physics, 00044 Frascati, Italy \\ 3 Department of Electrical and Computer Engineering, Michigan State University, \\ East Lansing, MI 48824, USA \\ * Correspondence: tamburrino@unicas.it
}

Received: 20 August 2019; Accepted: 2 October 2019; Published: 6 October 2019

Featured Application: efficient near-field analysis of cables illuminated by external fields.

\begin{abstract}
This paper proposes an efficient technique to solve the electromagnetic scattering problem, in the near zone of scatterers illuminated by external fields. The technique is based on a differential formulation of the Helmholtz equation discretized in terms of a finite element method (FEM). In order to numerically solve the problem, it is necessary to truncate the unbounded solution domain to obtain a bounded computational domain. This is usually done by defining fictitious boundaries where absorbing conditions are imposed, for example by applying the perfect matching layer (PML) approach. In this paper, these boundary conditions are expressed in an analytical form by using the Dirichlet-to-Neumann (DtN) operator. Compared to classical solutions such as PML, the proposed approach based on the DtN: (i) avoids the errors related to approximated boundary conditions; (ii) allows placing the boundary in close proximity to the scatterers, thus, reducing the solution domain to be meshed and the related computational cost; (iii) allows dealing with objects of arbitrary shapes and materials, since the shape of the boundary independent from those of the scatterers. Case-studies on problems related to the scattering from cable bundles demonstrate the accuracy and the computational advantage of the proposed technique, compared to existing ones.
\end{abstract}

Keywords: bundled cables; Dirichlet-to-Neumann map; electromagnetic compatibility; electromagnetic scattering; near-field analysis

\section{Introduction}

The near-field electromagnetic interaction between components has become an issue in high-frequency complex electronic systems, where it is highly likely to generate phenomena that affect the system performance, such as unwanted electromagnetic interference (EMI) or crosstalk noise. An accurate knowledge of the near-field distribution is mandatory in the design and verification of cable harnesses, integrated circuits, printed circuit boards, and Radio-Frequency (RF) systems. The near-field analysis allows, for instance, the location of hot spots and possible noise sources, or the estimation of the currents induced on the scatterers [1]. Specifically, facing both the emission and immunity issues related to unwanted scattering has become a major challenge for high-frequency systems in vehicles, aircraft, ships, and buildings. This problem poses many challenges both in experimental characterization (e.g., [2,3]) and in numerical modeling, usually based on full-wave or hybrid models (e.g., [4-8]). 
The study of the electromagnetic scattering from objects is a classical problem whose numerical solution can be obtained by using either integral or differential formulations [9-11]. In this paper, we focus on differential formulations solved by means of the finite element method (FEM). The final numerical model must be of course solved in a bounded solution domain, whereas the original problem is definite in an unbounded one (the whole space). Therefore, the unbounded domain must be truncated by an artificial boundary containing all the scatterers, and proper conditions must be imposed on such a boundary to avoid spurious reflections. Non-reflecting conditions can be classified into two main classes: non-reflective boundary conditions and non-reflective boundary layers. In the first class, the impinging waves are absorbed at the artificial boundary, as for instance when using the classical absorbing boundary conditions (ABC) [12]. In the second approach, the waves are absorbed in an extra layer, as in the case of perfectly matched layer (PML) [13]. A comprehensive review of lowand high-order non-reflecting boundary conditions may be found in [14].

The approximation introduced by the absorbing conditions is not the only issue when a scattering problem is solved in an artificially truncated domain: Another issue arises when a near-field analysis is required. Indeed, the absorbing conditions are usually matched on the structure of the far field, hence they are more efficient if the artificial boundary is placed in the far-field region, rather than in the near-field region. As a consequence, in order to achieve a given accuracy, the computational cost of a near-field simulation increases as the boundary is put closer to the scatterer.

These two issues may be solved if exact boundary conditions are imposed in the truncating boundary. This can be done by using the so-called Dirichlet-to-Neumann (DtN) operator on the artificial boundary. In this way, the "inner" problem (inside the artificial boundary) is decoupled from the "outer" one (outside the artificial boundary). Since the boundary conditions are exact, the boundary may be placed at any position, even in close proximity to the scattering object, thus reducing the computational domain (and hence the cost). This approach has been previously proposed for problems in acoustics, photonics, and electromagnetics, see [15-17]. Recently, it has been adopted for electromagnetic scattering problems applied to conducting cylinders [18], and composite materials [19]. An extension to the multiple scattering problems is also provided in [20]. The treatment of periodic structures, as those arising from arrays of conductors or Photonic Crystals, can be found in [21-24], based on the use of a semi-analytical DtN. A DtN-related approach can also be found in modeling atomic and molecular physics [25].

In this paper, the DtN approach is used to solve the scattering problem of a bundled cable illuminated by an external field. The authors have already presented initial results on single scatterers in the conference papers [26,27]. Here, we present the formulation in details, including the analysis of the structure of the matrices associated with the numerical formulation. In addition, the approach is here applied to analyze multiple scatterers, in case-studies of practical interest in the field of electromagnetic compatibility (EMC). Specifically, we efficiently evaluate the high-frequency near-field distribution, i.e., the solution in close proximity to the wire boundaries.

When arbitrary geometries are chosen for the boundary, the DtN operator can only be computed numerically, whereas it can be given in analytical form on canonical geometries such as the circle used in this paper. The numerical evaluation of the DtN may require a computational cost that severely limits its advantage. It is worth noting that the DtN only depends on the shape of the boundary but is not related to the shape of the scatterers: Therefore, scatterers of arbitrary shapes may be modeled by using the same DtN operator. The flexibility of the FEM is here combined with the efficiency of the DtN boundary conditions. In addition, thanks to the analytical form of the DtN for a circular boundary, the accuracy of the solution may be easily controlled by truncating the series at an appropriate term.

In addition, compared to existing approaches such as the boundary element method (BEM), where the DtN operator is implicitly imposed through an integral equation, the novelty of the approach proposed here resides in the use of an analytic form in terms of a series expansion.

The paper is organized as follows. Section 2 briefly recalls the formulation of the scattering problem in terms of Helmholtz equations. Then, the exact DtN operator is derived for a circular 
boundary and the numerical formulation of the problem is provided. In Section 3, first a benchmark problem is analyzed, to assess the method and evaluate the accuracy. Then the proposed technique is adopted to derive the high-frequency near-field distribution of the electrical field in a three-wire bundle configuration, under the action of an external field. The cases of wires in air or embedded in a dielectric are analyzed. A quantitative comparison between the DtN approach and the classical PML approach is provided.

\section{Methods}

\subsection{Scattering Problem and Helmholtz Equation}

The reference problem for this paper is depicted in Figure 1a, where an electromagnetic wave hits a number of objects, giving rise to a scattered field. The scatterers are dielectric but non-magnetic objects. Hereafter we assume that the scatterers are invariant along the $z$ axis.

At any spatial position, $r$, and at any time instant, $t$, the total field is given by the sum of the two aforementioned contributions:

$$
E(r, t)=E^{i}(r, t)+E^{S}(r, t) .
$$

The evaluation of the scattering from objects is a classic electromagnetic problem that may be formulated, for instance, by using Helmholtz equations [11]. To this end, in the following we consider time-harmonic fields,

$$
\boldsymbol{e}(\boldsymbol{r}, t)=\operatorname{Re}\left\{\boldsymbol{E}(\boldsymbol{r}) e^{j \omega t}\right\},
$$

where $\omega$ is the angular frequency. Hereafter, we assume $\mathrm{TM}_{\mathrm{z}}$ propagation, that is the incident wave is a Transverse Magnetic (TM) field with the electric field polarized along the $z$ axis and the scatterer is invariant in the $z$ direction. Therefore, the scattering problems reduces to a 2D scalar problem, where $E^{s}$ is the (scalar) unknown.

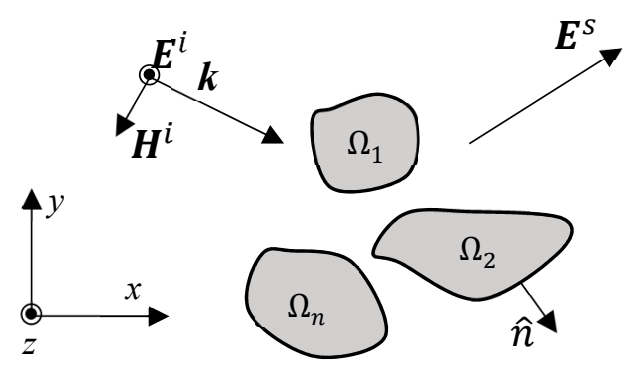

(a)

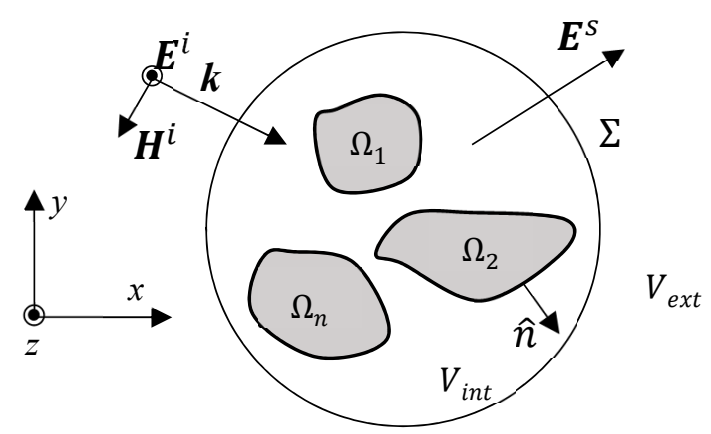

(b)

Figure 1. The analyzed scattering problem: $n$ arbitrarily shaped objects are illuminated by a known incident field. (a) References for the Helmholtz problem; (b) references for the Dirichlet-to-Neumann (DtN) formulation.

The scattered field is the solution of coupled Helmholtz equations written inside and outside the domain $\Omega$, defined as the union of all the scatterers $\Omega_{i}\left(\Omega=\Omega_{1} \cup \Omega_{2} \cup \ldots\right)$, see Figure 1a. Proper boundary conditions and regularity conditions at infinity must be imposed to close the problem. The final model is:

$$
\begin{gathered}
\nabla^{2} E+k^{2} m^{2} E=0, \text { in } \Omega, \\
\nabla^{2} E+k^{2} E=0, \text { in } R^{3} \backslash \Omega, \\
\left.E\right|_{\partial \Omega^{+}}-\left.E\right|_{\partial \Omega^{-}}=0, \\
\left.\partial_{n} E\right|_{\partial \Omega^{+}}-\left.\partial_{n} E\right|_{\partial \Omega^{-}}=0,
\end{gathered}
$$




$$
\lim _{r \rightarrow \infty} r^{1 / 2}\left(\partial_{n} E^{S}-j k E^{S}\right)=0 .
$$

Here, $k$ is the free-space wavenumber, $m(r)$ is the refraction index of the scatterers, and $\partial_{n}$ is the outward normal derivative. Specifically, conditions (5) and (6) enforce the continuity of the tangential components of the electrical and magnetic fields at the boundary of the scatterers, $\partial \Omega$, whereas (7) is the classical Sommerfeld radiation condition. It is worth noting that the problem (Equations (3)-(7)) is defined in an unbounded domain.

The numerical solution of the scattering problem (Equations (3)-(7)) may be obtained from integral or differential formulations $[10,11]$. The main advantage of using the integral formulations is the reduced solution domain, which includes only the material regions. Conversely, a weak point is the need of the Green function, which is available in analytical form only for simple geometries and material property distributions. In the general case, its numerical evaluation is extremely cumbersome because of the presence of singular or hyper-singular kernels. In addition, integral formulations give rise to a final numerical model characterized by fully populated matrices, which may pose challenging problems for storage and inversion.

Conversely, differential formulations do not require knowledge of the Green function and give rise to sparse matrices. However, a weak point is that the solution must be sought in the whole space. To cope with this problem, an artificial boundary, $\Sigma$, is usually introduced, in order to obtain a bounded solution domain, $V_{\text {int }}$, that includes all the scatterers (Figure $1 \mathrm{~b}$ ).

In propagation problems, the presence of this artificial boundary may affect the solution by introducing spurious non-physical reflections. To avoid these reflections, proper conditions must be imposed on it, to enable the absorption of such spurious waves. A state-of-the-art approach is the so-called, "perfectly matched layer" (PML), originally proposed in [13]. In the PML method, an extra layer of thickness, $\delta$, is added to the solution domain $B$ : In this extra layer, the wave is damped according to the following law:

$$
\exp \left[j(\omega t-k r)-\frac{k}{\omega} \int_{0}^{\delta} \sigma\left(r^{\prime}\right) d r \prime\right]
$$

where $\sigma(\cdot)$ is a positive function of the distance. In the PML approach, the position of the truncating boundary, $\Sigma$, and the layer thickness, $\delta$, are two degrees of freedom that must be adequately chosen to optimize the accuracy of the numerical solution.

\subsection{The Dirichlet-to-Neumann Operator}

The Dirichlet-to-Neumann (DtN) operator, $\Lambda(u)$, relates the values assumed by a scalar unknown function, $u$, on the boundary, $\Sigma$, (i.e., Dirichlet-type conditions), into the values of its normal derivative, $\partial_{n} u$, assumed on the same boundary, $\Sigma$ (i.e., Neumann-type conditions):

$$
\Lambda:\left.\left.u\right|_{\Sigma} \rightarrow \partial_{n} u\right|_{\Sigma}
$$

$u$ being the solution of the free-space Helmholtz equation outside $\Sigma$ (i.e., in the domain $V_{\text {ext }}$, see Figure $1 b$ ). By applying the DtN operator to $\Sigma$ it is possible to decouple the scattering problem (Equations (3)-(7)) into two problems: One problem defined in $V_{\text {in }}$ and another one defined in $V_{\text {ext }}$. In other words, the inner problem in $V_{\text {in }}$ can be closed exactly, without the need of introducing absorbing conditions or artificial absorbing layers.

In principle, the DtN may be numerically evaluated for any shape of the boundary. However, for canonical geometries it can be analytically evaluated. This entails better insight and more efficient numerical computations. Here we briefly recall its derivation for a circular boundary (details may be found in $[15,26,27])$. Introducing a polar coordinate system $(r, \theta)$ in the $x, y$ plane, the scalar wave equation can be solved in $V_{\text {ext }}$ by separation of variables as: 


$$
u(r, \theta)=\sum_{n=-\infty}^{\infty} U_{n} H_{n}^{(2)}(k r) e^{j n \theta}
$$

where $H_{n}^{(2)}$ is the Hankel function of second kind [28], and the coefficients $U_{n}$ are given by:

$$
U_{n}=\frac{1}{2 \pi H_{n}^{(2)}(k r)} \int_{0}^{2 \pi} u(r, \theta \prime) e^{-j n \theta \prime} d \theta \prime
$$

Note that the $U_{n}$ s do not depend on $r$. The DtN operator for a circular boundary $\Sigma$ of radius $R$ may be obtained by taking the normal derivative $\left(\partial_{n}=\partial_{r}\right)$ of $u$ at $r=R$. Thus, from Expressions (10) and (11) we have:

$$
\Lambda\left(\left.u\right|_{\Sigma}\right)=\frac{1}{2 \pi} \sum_{n=-\infty}^{\infty} \frac{H_{n}^{(2) \prime}(k R)}{H_{n}^{(2)}(k R)} \int_{0}^{2 \pi} u(R, \theta \prime) e^{-j n \theta^{\prime}} d \theta^{\prime},
$$

where $H_{n}^{(2) \prime}$ is the derivative of $H_{n}^{(2)}$ with respect to $r$, that can be also expressed as [28]:

$$
H_{n}^{(2) \prime}(k R)=\frac{H_{n-1}^{(2)}(k R)-H_{n+1}^{(2)}(k R)}{2} .
$$

The map is therefore known in analytical form, in terms of a series expansion.

It is worth noting that the position of the circular boundary may be arbitrarily chosen, even in close proximity to the scatterers. Therefore, the size of the solution domain may be strongly reduced, compared to the use of classical absorbing conditions such as PML, that are more efficient when the boundary is placed in the far-field region.

\subsection{Numerical Model}

In order to numerically solve the scattering problem in the solution domain $\mathrm{V}_{\text {int }}$, Equations (3) and (4) are cast in weak form as:

$$
\int_{\Sigma} \varphi \Lambda\left(E^{S}\right) d l-\int_{\mathrm{V}_{\text {int }}}\left[\nabla \varphi \cdot \nabla E^{S}-k^{2} m^{2} \varphi E^{s}\right] d S=f,
$$

where $f$ is a known term depending on the external impinging field:

$$
f=-k^{2} \int_{\mathrm{V}_{\text {int }}}\left(m^{2}-1\right) \varphi E^{i} d S,
$$

and Equations (14) and (15) hold for any function $\varphi \in H^{1}\left(\mathrm{~V}_{\text {int }}\right)$, for $E^{s} \in H^{1}\left(\mathrm{~V}_{\text {int }}\right)$, where $H^{1}\left(\mathrm{~V}_{\text {int }}\right)$ is the Sobolev space defined as $H^{1}\left(\mathrm{~V}_{\text {int }}\right)=\left\{\varphi \in L^{2}\left(\mathrm{~V}_{\text {int }}\right): \nabla \varphi \in L^{2}\left(\mathrm{~V}_{\text {int }}\right)\right\}$, [29].

To obtain the discrete model, first we express the unknown as $E^{s}(x, y)=\sum_{i=1}^{N} c_{i} \varphi_{i}(x, y)$, where the shape functions are first-order isoparametric nodal elements, the $c_{i}$ s are the unknown coefficients (degrees of freedom, DoF) and $N$ is the total number of nodes of the finite element mesh. Then, the Galerkin approach applied to Equation (14) gives the following linear system of equations for the DoFs:

$$
(\mathbf{\Lambda}+\mathbf{A}) c=f,
$$

where

$$
\Lambda_{l k}=\int_{\Sigma} \varphi_{l} \Lambda\left(\varphi_{k}\right) d l, \mathrm{~A}_{l k}=-\int_{\mathrm{V}_{\text {int }}}\left[\nabla \varphi_{l} \cdot \nabla \varphi_{k}-k^{2} m^{2} \varphi_{l} \varphi_{k}\right] d S,
$$




$$
f_{l}=-k^{2} \int_{\mathrm{V}_{\text {int }}}\left(m^{2}-1\right) \varphi_{l} E^{i} d S .
$$

It is convenient partitioning the unknowns DoFs in $c_{b}$ and $c_{i}$ which are related to the boundary and the internal nodes of the finite element mesh. In this way, we get:

$$
\left(\left[\begin{array}{cc}
\Lambda_{b b} & 0 \\
0 & 0
\end{array}\right]+\left[\begin{array}{cc}
A_{b b} & A_{b i} \\
A_{i b} & A_{i i}
\end{array}\right]\right)\left[\begin{array}{c}
c_{b} \\
c_{i}
\end{array}\right]=\left[\begin{array}{c}
f_{b} \\
f_{i}
\end{array}\right]
$$

We notice that $\boldsymbol{\Lambda}_{b b}$ is a $N_{b} \times N_{b}$ fully populated matrix, whereas A is a $N \times N$ sparse matrix, where $N_{b}$ is the number of boundary nodes, usually much smaller than $N\left(N_{i}\right)$. Matrix $\boldsymbol{A}$ is the classical stiffness matrix arising from FEM.

For solving the linear system (19), an iterative method is preferable to a direct method. In doing this, there are several key aspects such as memory occupation and computational cost for a matrix-by-vector product. The memory required to store $\Lambda$ and $\mathbf{A}$ is $N_{b}^{2}$ and $O(N)$, respectively. On the other hand, the number of multiplications for a matrix-by-vector product is $N_{b}^{2}+O(N)$, which can be further reduced by properly treating the stiffness matrix. To make a comparison with the PML, we notice that the memory occupation and the number of multiplications is $O\left(N_{\delta}\right)+O\left(N_{P M L}\right)$, where $N_{P M L}$ are the number of DoFs in the computational domain for the PML and $N_{\delta}$ the number of DOFs located in the absorbing layer of thickness $\delta$, see Equation (8). As mentioned, the computational domain for the PML has to be far from the scatterer, thus it should be $N_{P M L} \gg N$, for a prescribed mesh density. Regarding iterative methods, in this work we applied the biconjugate gradient technique [30], with an Incomplete Lower-Upper (ILU) factorization used as a preconditioner [31].

In conclusion, at a given accuracy, the DtN approach, if compared to the PML one, does not introduce extra DoFs in the PML layer $\left(N_{\delta}\right)$ and can strongly reduce the inner DoFs $\left(N_{i}\right)$, since the boundary of the computational domain can be placed close to the scatterers. Conversely, the DtN approach introduces the (once for all) computational costs related to the assembly of matrix (Equation (17)) and to the matrix-by-vector product related to $\boldsymbol{\Lambda}_{b b}$.

\section{Results}

\subsection{Case Study 1: Scattering from a Perfect Conductor Cylinder}

The first case study is a benchmark case that is a detailed analysis of an example provided in [26]: A perfectly conducting cylinder, infinitely long along $z$, is illuminated by a $\mathrm{TM}_{z}$ plane wave, with wave-vector, $\mathbf{k}$, oriented along the $x$ axis (see Figure 2):

$$
E^{i}(r, \theta)=E_{0} e^{-j k r \cos \theta} .
$$

For this problem, the scattering field may be given in closed form [26] as:

$$
E^{S}(r, \theta)=\sum_{n=-\infty}^{\infty} w_{n} H_{n}^{(2)}(k r) e^{j n \theta},
$$

where coefficients $w_{n}$ are given by:

$$
w_{n}=-j^{-n} \frac{J_{n}(k a)}{H_{n}^{(2)}(k a)} E_{0} .
$$

For this case study, a frequency of $3 \mathrm{GHz}$ was chosen for the exciting wave, corresponding to a free-space wavelength $\lambda=10 \mathrm{~cm}$. A cylinder of radius $a=\lambda$ was assumed. The distribution of the scattered field, evaluated as in Expansion (21), is shown in Figure 3 and will be taken in the following as the reference solution. 
In implementing the DtN solution, a circular boundary, $\Sigma$, of radius $R=1.2 \lambda$ was chosen, therefore locating the boundary of the computational domain in close proximity to the scatterer, in the near-field zone. The finite element mesh was made by triangular elements. The number of the degrees of freedom corresponds to the number, $N$, of mesh nodes.

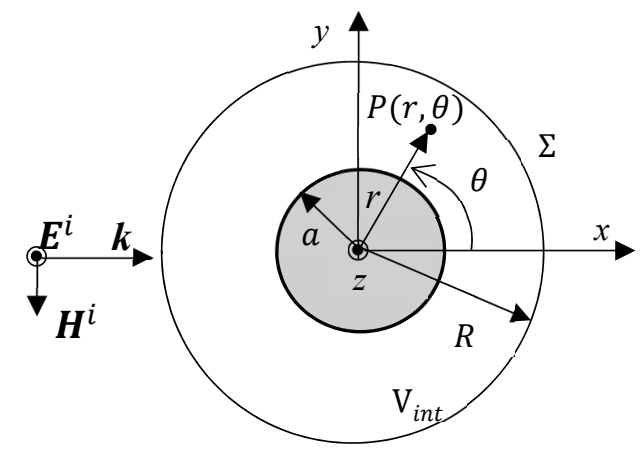

(a)

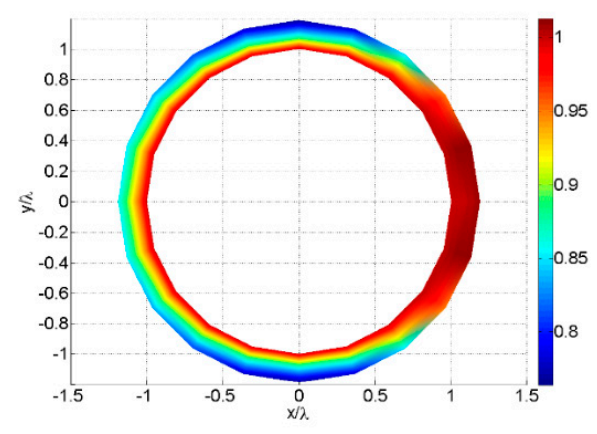

(b)

Figure 2. Case study 1. (a) Geometry and references for evaluating the scattering from an infinitely long conducting cylinder, illuminated by a $\mathrm{TM}_{\mathrm{z}}$ plane wave. (b) Map of the amplitude of the scattered field, normalized to that of the incident field. The axes $x$ and $y$ are normalized to the wavelength value [26].

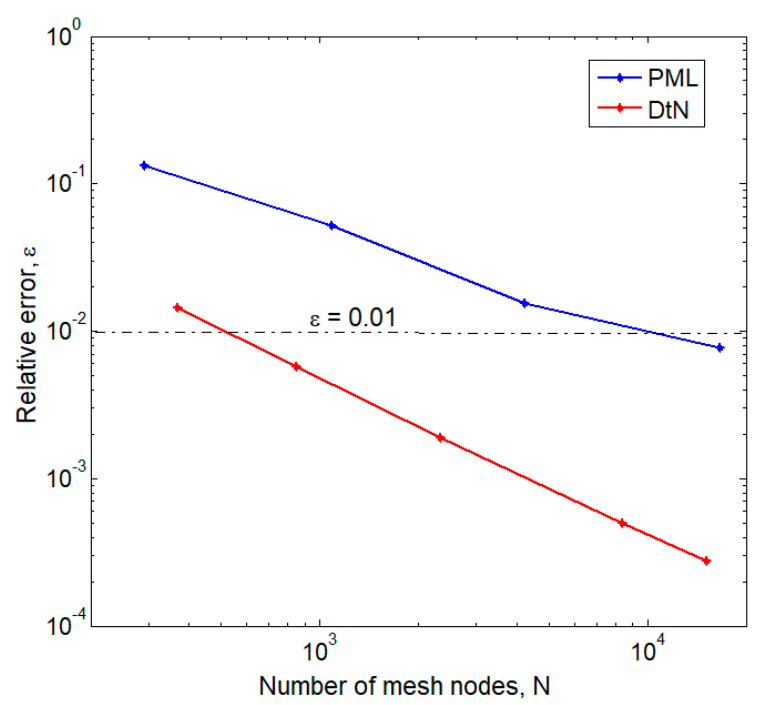

Figure 3. Case study 1 (perfectly conducting cylinder). Relative error as a function of the number of mesh nodes, for the DtN and perfect matching layer (PML) approaches. The PML solution refers to the optimized value of the layer thickness, $\delta=1.4 \lambda$.

The PML approach is instead implemented by using a commercial simulator (COMSOL Multiphysics $($, [32]), which requires a layer of thickness, $\delta$, to be added externally, as pointed out. Such a thickness is here chosen in an optimal way, as discussed in the following.

The DtN and PML numerical solutions are compared in terms of the relative error with respect to the reference solution, defined by using norm-2:

$$
\varepsilon \equiv \frac{\left\|E_{n}^{s}-E_{r}^{s}\right\|}{\left\|E_{r}^{s}\right\|},
$$

where the subscripts " $n$ " and " $r$ " indicate the numerical and the reference solutions, respectively. The numerical solutions can be obtained by using the DtN or the PML approaches. In the latter case, the layer thickness, $\delta$, is set to a value that optimizes the curve of the performances in terms of the error (23). In this case, this value was found to be $\delta=1.4 \lambda$. 
The errors are plotted in Figure 3, versus the number, $N$, of mesh nodes, which is related to the computational cost, as pointed out before. The result validates the proposed DtN-based numerical scheme and clearly indicates its advantage with respect to the PML approach. Indeed, for a given value of $N$ the relative error for $\mathrm{DtN}$ is about one order of magnitude smaller. Conversely, for a fixed accuracy, the DtN approach requires much less elements: For instance, a relative error $\varepsilon=0.01$ would be obtained with $N=501$ for DtN and $N=11,305$ for PML, hence with a reduction of the DoFs of about $23 \times$. Similar performances may be observed in the case of a single penetrable cylinder, or for arbitrarily shaped scatterers, as shown in [27].

\subsection{Case Study 2: Scattering from a Cable Bundle in Air}

As pointed out in the introduction, the study of the near-field scattering from unshielded bundled cables is of great interest for the EMC analysis of a wide variety of electronic applications, from automotive on-board systems to buried power lines. Here, we refer to the geometry shown in Figure $4 a$, where three conducting wires are bundled in an unshielded cable, surrounded by air $\left(\varepsilon_{r}=1\right)$. The frequency is again assumed to be equal to $3 \mathrm{GHz}$, with the wire radius $a=\lambda / 6$, and the cable radius $b=\lambda$. The three wires are reciprocally rotated by $120^{\circ}$ and their centers are placed at a distance equal to $c=\lambda / 2$ from the cable center.

The DtN solution is calculated by choosing again a circular boundary of radius $R=1.2 \lambda$. Here, the reference solution is given by the numerical solution provided by commercial code COMSOL Multiphysics $₫$ with PML approach, stabilized to an accuracy below $10^{-4}$. For this example, this requires about $1.1 \times 10^{6}$ mesh elements. The obtained distribution of the amplitude of the scattered field is plotted in Figure $4 \mathrm{~b}$.

The evaluation of the maximum scattered field gives an important information in EMC analysis, since it could provide the worst-case estimation of unwanted radiated emission and/or crosstalk noise between the conductors in the cable. Therefore, in the following we study the maximum value of the scattered field in the near-field region, namely inside the circle of radius $R=1.2 \lambda$. According to the solution in Figure $5 b$, the maximum value of the scattered field, normalized to the amplitude of the incident wave, is equal to $\left|E^{S}\right| / E_{0}=1.604$. In Figure 5a we compare the DtN and PML numerical solution for increasing value number, $N$, of the mesh nodes: The DtN solution converges to the reference value much faster than the PML one; the required values of $N$ for reaching a difference of $1 \%$ between the numerical solutions and the reference one are reported in Table 1. The use of the DtN provides a reduction in $N$ of a factor of about 5 .

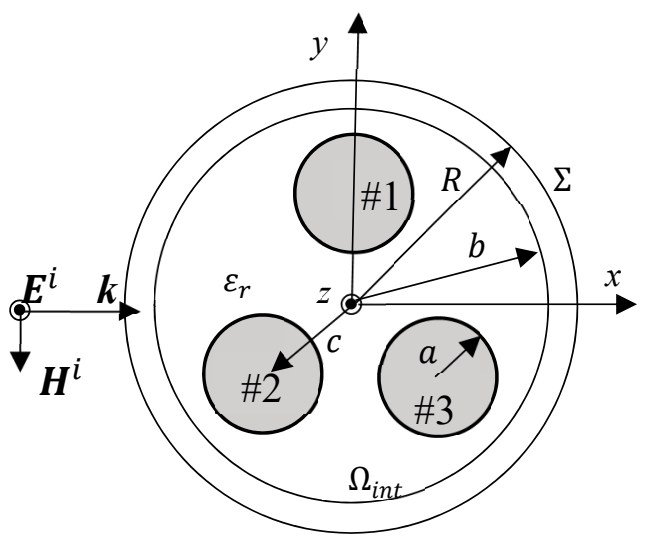

(a)

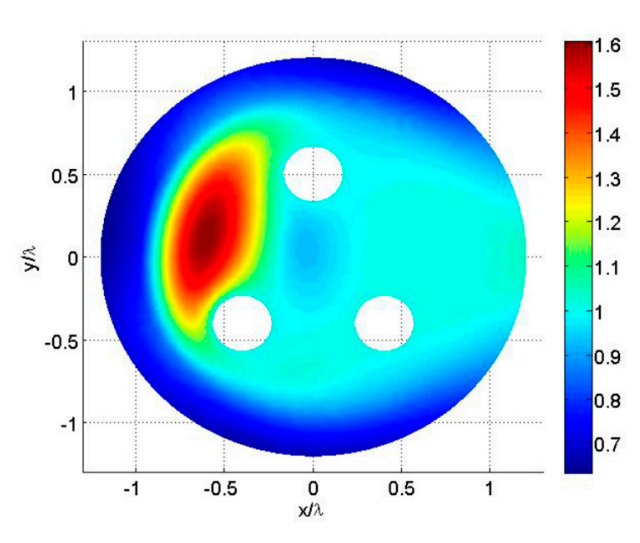

(b)

Figure 4. Case study 2 (cable bundle in air). (a) Geometry and references for evaluating the scattering from the cable, illuminated by a $\mathrm{TM}_{\mathrm{z}}$ plane wave; (b) Map of the amplitude of the scattered field, normalized to that of the incident field. The axes $x$ and $y$ are normalized to the wavelength value. 
Table 1. Required number of mesh nodes for $1 \%$ accuracy, Case study 3.

\begin{tabular}{ccc}
\hline Case & DtN & PML \\
\hline w/o dielectric & 511 & 2638 \\
\hline w dielectric & 11,573 & 59,534 \\
\hline
\end{tabular}

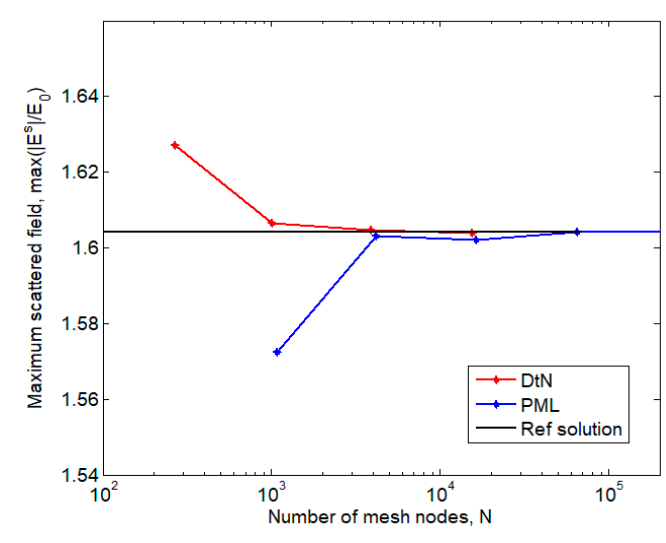

(a)



(b)

Figure 5. Case study 2 (cable bundle in air). (a) Maximum value of the scattered field, normalized to amplitude of the incident field, versus the number of mesh nodes: The DtN and PML solutions are compared to the reference one. (b) Condition number of the stiffness matrices associated with the two numerical solutions, versus the number of mesh nodes.

Finally, in order to better estimate the gain in terms of computational cost, beside the number of the mesh nodes, $N$, it is of interest to compare the condition number, $k$, of the resulting stiffness matrix $M$ associated with the two numerical models, defined in our case as:

$$
k(\boldsymbol{M}) \equiv\|\boldsymbol{M}\|\left\|\boldsymbol{M}^{-1}\right\|,
$$

where $\boldsymbol{M}$ is the 2-norm of $\boldsymbol{M}$. Indeed, the computational cost of the numerical solution (the number of iterations required for convergence in an iterative solver), is inversely related to the condition number. In Figure $6 \mathrm{~b}$, the condition number, $k$, versus the number of the mesh nodes, $N$, is reported. The DtN scheme provides a lower condition number, hence, outperforming the PML one also in this sense.

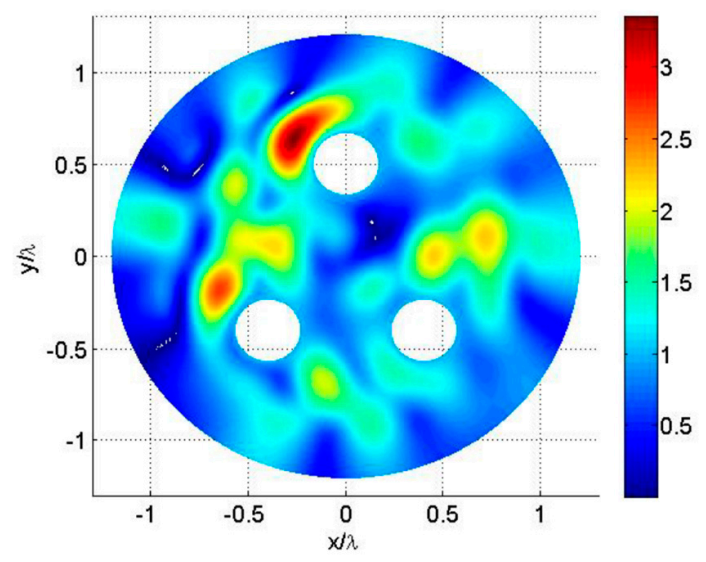

(a)

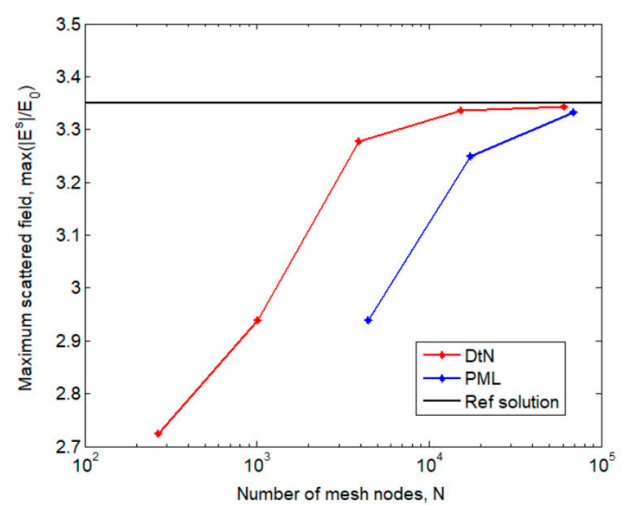

(b)

Figure 6. Case study 3 (cable bundle with dielectric). (a) Map of the amplitude of the scattered field, normalized to that of the incident field. The axes $x$ and $y$ are normalized to the wavelength value. (b) Maximum value of the scattered field, normalized to amplitude of the incident field, versus the number of mesh nodes: The DtN and PML solutions are compared to the reference one. 


\subsection{Case Study 3: Scattering from a Cable Bundle in a Dielectric Coating}

The last example refers to the same problem as Case study 2, but assuming the wires surrounded by a dielectric of relative permittivity equal to $\varepsilon_{r}=4$. The spatial distribution of the scattered field is now given in Figure 6a, giving a maximum value of the scattered field, normalized to the amplitude of the incident wave, equal to $\left|E^{S}\right| / E_{0}=3.3505$. The reference solution is obtained by using COMSOL Multiphysics $囚$ with an accuracy below $10^{-4}$. In this example, this requires about $16.5 \times 10^{6}$ mesh elements.

In Figure 6b, we compare the DtN and PML numerical solution for increasing value number, $N$, of the mesh nodes: Once again, the DtN solution converges to the reference value much faster than the PML one; the required values of $N$ for an accuracy of $1 \%$ reported in Table 1 indicates now a gain in using the DtN of a factor $5 \times$.

In Figure 7, the condition number $k$ defined as in Expression (24) is reported, highlighting a similar behavior compared to Case study 2.

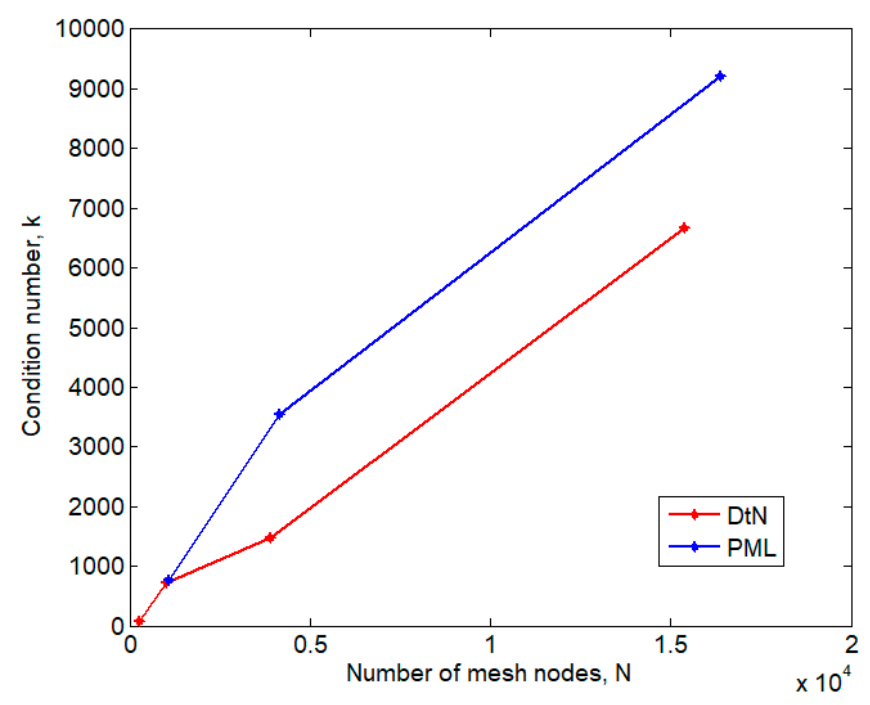

Figure 7. Case study 3 (cable bundle with dielectric). Condition number of the stiffness matrices associated to the two numerical solutions versus the number of mesh nodes.

\section{Conclusions}

The use of the Dirichlet-to-Neumann (DtN) operator has been, here, shown to be a suitable way to efficiently evaluate the near-zone electromagnetic field in a scattering problem, when such a problem is numerically solved by means of differential formulations, discretized with the finite elements method. Indeed, thanks to the DtN operator, the numerical problem is cast in a bounded computational domain where the boundary conditions are imposed exactly, with analytical expressions. Specifically, in this paper the DtN map was provided with reference to a circular boundary, in terms of a series expansion of Henkel functions. Given its features, the proposed approach may be applied to scatterers with arbitrary shapes, topology, and material properties.

The analyzed case-studies, referring to bundles of conducting wires with or without dielectrics, compare the DtN approach to the state-of-art absorbing boundary condition, i.e., the perfect matching layer (PML). For a given accuracy, the DtN solution requires a lower number of mesh nodes compared to the PML one: For instance, for a fixed maximum error of $1 \%$, the mesh nodes are reduced by a factor of 5. In addition, for a given number of mesh nodes, the conditioning number of the stiffness matrix associated to the DtN is definitely better than that related to the PML solution. Both these results lead to a lower computational cost.

Author Contributions: Conceptualization, A.T.; methodology, A.M.P., A.T., A.M, S.V.; software and validation, A.M.P., S.V.; investigation, S.V.; writing, A.M.P., A.M.; supervision, A.T. 
Funding: The work was partially supported by the Project: "Smart Distributed Systems", funded by MIUR, Italian Ministry of University and Research, under the program "Dipartimenti di Eccellenza 2018-2022".

Conflicts of Interest: The authors declare no conflict of interest.

\section{References}

1. Mikki, S.; Antar, Y. New Foundations for Applied Electromagnetics: The Spatial Structure of Fields; Artech House: Boston, MA, USA, 2016.

2. Weng, H.; Beetner, D.G.; DuBroff, R.E. Prediction of radiated emissions using near-field measurements. IEEE Trans. Electromagn. Compat. 2011, 53, 891-899. [CrossRef]

3. Deschrijver, D.; Vanhee, F.; Pissoort, D.; Dhaene, T. Automated Near-Field Scanning Algorithm for the EMC Analysis of Electronic Devices. IEEE Trans. Electromagn. Compat. 2011, 54, 502-510. [CrossRef]

4. Baudry, D.; Arcambal, C.; Louis, A.; Mazari, B.; Eudeline, P. Applications of the Near-Field Techniques in EMC Investigations. IEEE Trans. Electrom. Comp. 2007, 49, 485-493. [CrossRef]

5. Clavel, E.; Tran, T.S.; Aimé, J.; Meunier, G.; Roudet, J. Far Field Extrapolation from Near Field Interactions and Shielding Influence Investigations Based on a FE-PEEC Coupling Method. Electronics. 2013, 2, 80-93. [CrossRef]

6. Liu, D.; Wang, Y.; Kautz, R.W.; Altunyurt, N.; Chandra, S.; Fan, J. Accurate Evaluation of Field Interactions Between Cable Harness and Vehicle Body by a Multiple Scattering Method. IEEE Trans. Electrom. Comp. 2017, 59, 383-393. [CrossRef]

7. Capriglione, D.; Chiariello, A.G.; Maffucci, A. Accurate Models for Evaluating the Direct Conducted and Radiated Emissions from Integrated Circuits. Appl. Sci. 2018, 8, 477. [CrossRef]

8. Maffucci, A.; Miano, G.; Villone, F. Full-wave transmission-line theory. IEEE Trans. Magn. 2003, 39, $1594-1597$. [CrossRef]

9. Bello, M.; Liu, J.; Guo, R. Three-Dimensional Wide-Band Electromagnetic Forward Modelling Using Potential Technique. Appl. Sci. 2019, 9, 1328. [CrossRef]

10. Rao, S.; Wilton, D.; Glisson, A. Electromagnetic scattering by surfaces of arbitrary shape. IEEE Trans. Antennas Prop. 1982, 30, 409-418. [CrossRef]

11. Glisson, A. An integral equation for electromagnetic scattering from homogeneous dielectric bodies. IEEE Trans. Antennas Prop. 1984, 32, 173-175. [CrossRef]

12. Engquist, B.; Majda, A. Absorbing Boundary Conditions for the Numerical Simulation of Waves. Math. Comp. 1977, 31, 629-651. [CrossRef]

13. Berenger, J.P. A Perfectly Matched Layer for the absorption of electromagnetic waves. J. Comput. Phys. 1994, 114, 185-200. [CrossRef]

14. Grote, M.J. Non-reflecting boundary conditions for electromagnetic scattering. Int. J. Numer. Model. 2000, 13, 397-416. [CrossRef]

15. Keller, J.B.; Givoli, D. Exact non-reflecting boundary conditions. J. Comput. Phys. 1989, 82, 172-192. [CrossRef]

16. Keller, J.B.; Givoli, D. Numerical Methods for Problems in Infinite Domains; Elsevier: Amsterdam, The Netherlands, 1992.

17. Harari, I.; Hughes, T.J.R. Studies of domain-based formulations for computing exterior problems of acoustics. Int. J. Numer. Methods Eng. 1994, 37, 2935-2950. [CrossRef]

18. Baratta, I.A.; Silva, E.J. Multi-Domain Transmission Conditions for Domain Decomposition Methods Applied to Scattering Problems. IEEE Trans. Magn. 2017, 54. [CrossRef]

19. Milton, G.; Cassier, M.; Mattei, O.; Milgrom, M.; Welters, A. Extending the Theory of Composites to Other Areas of Science; Milton-Patton Publishing: Salt Lake City, UT, USA, 2016.

20. Grote, M.J.; Kirsch, C. Dirichlet-to-Neumann Boundary Conditions for Multiple Scattering Problems. J. Comput. Phys. 2004, 201, 630-650. [CrossRef]

21. Huang, Y.; Lu, Y.Y. Scattering from Periodic Arrays of Cylinders by Dirichlet-to-Neumann Maps. J. Lightw. Technol. 2006, 24, 3448-3453. [CrossRef]

22. Wu, Y.; Lu, Y.Y. Dirichlet-to-Neumann map method for analyzing periodic arrays of cylinders with oblique incident waves. J. Opt. Soc. Am. B 2009, 26, 1442-1449. [CrossRef]

23. Wu, Y.; Lu, Y.Y. Dirichlet-to-Neumann map method for analyzing interpenetrating cylinder arrays in a triangular lattice. J. Opt. Soc. Am. B 2008, 25, 1466-1473. [CrossRef] 
24. She, S.; Lu, Y.Y. Improved Dirichlet-to-Neumann map method for scattering by circular cylinders on a lattice. J. Opt. Soc. Am. A 2012, 29, 1999-2004. [CrossRef] [PubMed]

25. Tennyson, J. Electron-molecule collision calculations using the R-matrix method. Phys. Rep. 2010, 491, $29-76$. [CrossRef]

26. Maffucci, A.; Perrotta, A.; Rubinacci, G.; Tamburrino, A.; Ventre, S. Efficient numerical evaluation of the electromagnetic scattering from arbitrarily-shaped objects by using the Dirichlet-to-Neumann map. In Proceedings of the 2017 International Conference on Electromagnetic in Advanced Applications (ICEAA), Verona, Italy, 11-15 September 2017; pp. 1616-1619.

27. Maffucci, A.; Perrotta, A.; Ventre, S.; Tamburrino, A. Numerical solution of electromagnetic scattering problems based on the Dirichlet-to-Neumann map. In Proceedings of the 2018 IEEE 4th Int. Forum on research and technology for Society and Industry (RTSI), Palermo, Italy, 10-13 September 2018; pp. 298-302.

28. Abramowitz, M.; Stegun, I. Handbook of Mathematical Functions; National Bureau of Standards: Washington, DC, USA, 1964.

29. Evans, L.C. Partial Differential Equations; American Mathematical Society, Graduate studies in Mathematics: Providence, RI, USA, 2010.

30. Van der Vors t, H.A. Bi-CGSTAB: A Fast and Smoothly Converging Variant of Bi-CG for the Solution of Nonsymmetric Linear Systems. SIAM J. Sci. Stat. Comput. 1992, 13, 631-644. [CrossRef]

31. Saad, Y. Iterative Methods for Sparse Linear Systems; PWS Publishing Company: Boston, MA, USA, 1996.

32. COMSOL Multiphysics $®$, Modeling Software. Available online: www.comsol.com/products (accessed on 10 May 2019).

(C) 2019 by the authors. Licensee MDPI, Basel, Switzerland. This article is an open access article distributed under the terms and conditions of the Creative Commons Attribution (CC BY) license (http://creativecommons.org/licenses/by/4.0/). 